\title{
The occultation events of the Herbig Ae/Be star V1247 Orionis ${ }^{\star}$
}

\author{
J. A. Caballero ${ }^{1,2}$ \\ 1 Centro de Astrobiología (CSIC-INTA), Carretera de Ajalvir km 4, 28850 Torrejón de Ardoz, Madrid, Spain \\ e-mail: caballero@astrax.fis.ucm.es \\ 2 Departamento de Astrofísica y Ciencias de la Atmósfera, Facultad de Física, Universidad Complutense de Madrid, \\ 28040 Madrid, Spain \\ Received 7 January 2010 / Accepted 16 February 2010
}

ABSTRACT

\begin{abstract}
Aims. I study new deep ( $\Delta V \approx 1.20-1.65 \mathrm{mag})$ occultation events of the $\delta$ Scuti, Herbig Ae/Be star V1247 Ori in the Ori OB1 b association.

Methods. I use the $V$-band ASAS light curve of V1247 Ori, which covers the last nine years, together with photometric data in the near-ultraviolet, visible, near-, and far-infrared taken from the literature. I carry out a periodogram analysis of the "cleaned" light curve and construct the spectral energy distribution of the star.

Results. The star V1247 Ori is interesting for the study of the UX Orionis phenomenon, in which Herbig Ae/Be stars are occulted by their protoplanetary discs, for three reasons: brightness $(V \approx 9.85 \mathrm{mag})$, large infrared excess at $20-100 \mu \mathrm{m}\left(F_{60} \approx 10 \mathrm{Jy}\right)$, and photometric stability out of occultation $(\sigma(V) \approx 0.02 \mathrm{mag})$, which may help to determine the location and spatial structure of the occulting disc clumps.
\end{abstract}

Key words. astronomical databases: miscellaneous - protoplanetary disks - stars: individual: V1247 Ori - stars: pre-main sequence - stars: variables: $\delta$ Scuti - stars: variables: T Tauri, Herbig Ae/Be

\section{Introduction}

With the aim of finding highly variable stars of the Orion Belt not listed by Caballero et al. (2010), I explored the All Sky Automated Survey ASAS-3 Photometric $V$-band Catalogue of variable stars of Pojmański (2002). Among the light curves of variable ASAS stars unidentified by Caballero et al. (2010), the one of the pre-main sequence star V1247 Ori stood out because of at least two unnoticed deep occultation events, one of which lasted for about $20 \mathrm{~d}$ (Fig. 1). Apart from that, the light curve of V1247 Ori is highly stable, with reported light curve standard deviations of only $0.010-0.013$ mag (see below).

The star V1247 Ori (HD 290764) ${ }^{1}$ was classified as an intermediate A-type giant in the Orion "ring" by Schild \& Cowley (1971). Later, Guetter (1981) assigned it membership in the Warren \& Hesser (1977)'s subgroup b2, which is now known as the central part of the Ori OB1 b association, in the vicinity of the bright supergiant Alnilam ( $\epsilon$ Ori, $\tau \sim 5-10 \mathrm{Ma}-$ Caballero \& Solano 2008). MacConnell (1982) discovered H $\alpha$ emission with an estimated densitity 2 ("on a scale from T, trace, to 5") in an optical spectrum of V1247 Ori. Later, Vieira et al. (2003) identified an "H $\alpha$ symmetric profile without, or with only very shallow, absorption features", but found no forbidden lines. Wouterloot \& Walmsley (1986) firstly identified the star with the infrared source IRAS 05355-0117 and provided a restrictive upper limit to the presence of $\mathrm{H}_{2} \mathrm{O}$ radio lines. Next, Wouterloot et al. $(1988,1989)$ did the same for $\mathrm{NH}_{3}$ and

* Figure 4 is only available in electronic form at http://www . aanda. org

1 In some works, V1247 Ori is incorrectly listed as BD-01 983 (e.g., Kazarotevs 1993; Rodríguez et al. 1994), but that is the B8 star HD 290765, and as vdB 50 (Vieira et al. 2003), but that is the B3Vn star HD 37674.

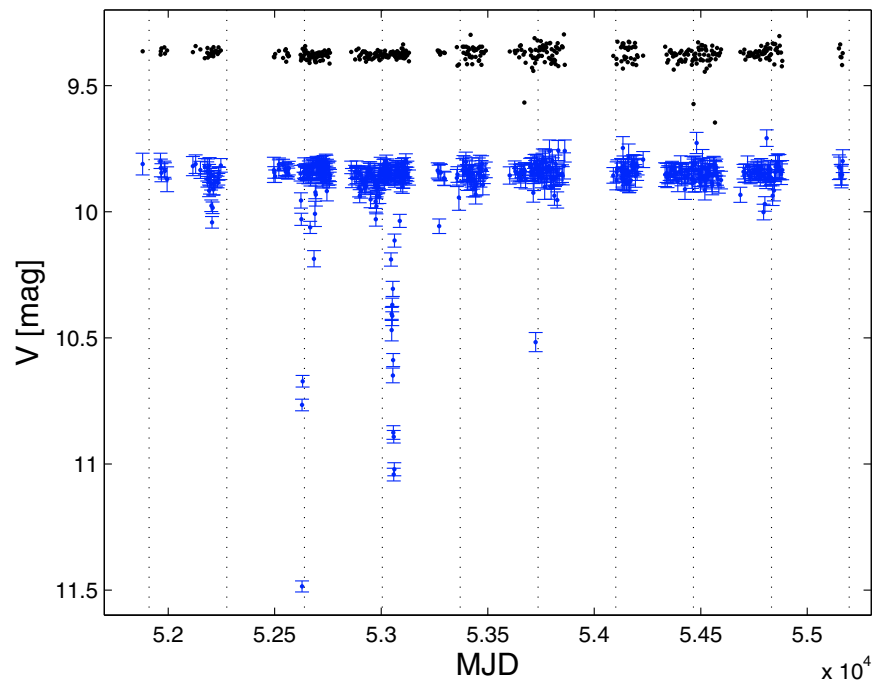

Fig. 1. ASAS light curves of V1247 Ori (bottom, [blue] error bars) and a nearby, slightly brighter, field late-K star for comparison (HD 290760 , $\rho \sim 19$ arcmin; top, [black] dots). Vertical dotted lines indicate the first day of the years 2001 to 2010 . The two main occultations events in V1247 Ori occurred in 2002 Dec. $\left(V_{\min } \approx 11.50 \mathrm{mag}\right)$ and 2004 Feb. $\left(V_{\min } \approx 11.05 \mathrm{mag}\right)$. Note the seasonal gaps.

CO radio lines. The spectral energy distribution (SED) of V1247 Ori shows clear flux excess from the $J$ band to 60 $100 \mu \mathrm{m}$ (Fujii et al. 2002; Clarke et al. 2005; Caballero \& Solano 2008), and is composed of two components, one warm (1.2$2.2 \mu \mathrm{m})$ and the other cool $(20-100 \mu \mathrm{m})$. It also displays intrinsic polarisation $\left(P_{\mathrm{pol}}=0.32 \pm 0.04 \%, \theta_{\mathrm{pol}}=52.9 \mathrm{deg}\right)$, which indicates that its circumstellar envelope is not spherical (Rodrigues et al. 2009). Taking into account all these factors, V1247 Ori has 
Table 1. $V$ magnitudes of V1247 Ori published in the literature.

\begin{tabular}{cccc}
\hline \hline $\begin{array}{c}V \\
{[\mathrm{mag}]}\end{array}$ & Ref. $^{a}$ & $\begin{array}{c}V \\
{[\mathrm{mag}]}\end{array}$ & Ref. $^{a}$ \\
\hline $9.88 \pm 0.01:$ & Sh62 & $9.82 \pm 0.03$ & TYC \\
$9.854 \pm 0.007$ & Ru76 & $9.87 \pm 0.02$ & Ri00 \\
$9.846 \pm 0.013$ & Ru88 & $9.81 \pm 0.05$ & Fu02 \\
$9.844 \pm 0.010$ & LR92 & $9.88 \pm 0.01:$ & Vi03 \\
$9.90 \pm 0.01$ & Eg92 & $9.85 \pm 0.13$ & Dr06 \\
$9.92 \pm 0.01:$ & Ha99 & & \\
\hline
\end{tabular}

References. ${ }^{(a)}$ Sh62: Sharpless (1962); Ru76: Rufener (1976); Ru88: Rufener (1988); LR92: Lampens \& Rufener (1992); Eg92: Egret et al. (1992); Ha99: Handler (1999); TYC: Høg et al. (2000), transformed from $B_{T} V_{T}$ using the expression $V=V_{T}-0.090\left(B_{T}-V_{T}\right)$; Ri00: Richmond et al. (2000); Fu02: Fujii et al. (2002); Vi03: Vieira et al. (2003); Dr06: Droege et al. (2006).

been classified as a Herbig Ae/Be star with a circumstellar disc (Herbig Ae/Be stars are the high-mass "counterparts" of T Tauri stars - Wouterloot \& Walmsley 1986; García-Lario et al. 1997; Fujii et al. 2002; Vieira et al. 2003).

Lampens \& Rufener (1982) repeatedly observed V1247 Ori on 189 occasions during 67 days in early 1984 and another 18 times on a night about one year later, probably on 1984 Dec. 03. They indicated that the measurements made on that night were fainter by 0.03 mag compared to the rest of the data. They found a period of photometric variability $P=$ $0.096967 \mathrm{~d}$ with an amplitude of only $0.016 \pm 0.008 \mathrm{mag}$, and classified it as a $\delta$ Scuti star. Afterwards, V1247 Ori has appeared in a number of catalogues of such kind of pulsators (e.g., García et al. 1995; Rodríguez et al. 2000). Furthermore, it is one of the few known pre-main-sequence $\delta$ Scuti stars (Zwintz 2008). The actual period of photometric variability may slightly differ from the Lampens \& Rufener (1982) value, since Debosscher et al. (2007) tabulated a different period at $P \approx 0.089973 \mathrm{~d}$. Besides, the authors provided a second period at $P \approx 75.255 \mathrm{~d}$. In any case, the measured amplitudes are very low, and the $V$ magnitude of V1247 Ori has never been reported to vary more than $0.05 \mathrm{mag}$. However, the occultation events in the ASAS light curve, which may be related to an edge-on circumstellar disc, were deeper than $1 \mathrm{mag}$.

\section{Analysis and results}

In Table 2 I compiled coordinates, proper motions, magnitudes and fluxes (from the near-ultraviolet $U$ band to the IRAS $100 \mu \mathrm{m}$ one), and spectral types of V1247 Ori from the literature. Apart from the spectral type determinations by Schild \& Cowley (1971) and Vieira et al. (2003), there have been other measurements at A2 by Heckmann (1975 - taken from the Publications of the Leander McCormick Observatory) and A7 by Nesterov et al. (1995). I tabulated neither the magnitudes $r^{\prime}$ from the Carlsberg Meridian Catalogue 14 (CMC14; Carlsberg Meridian Telescope 2006) and $i^{\prime}$ from the Deep Near Infrared Survey of the Southern Sky (DENIS; Epchtein et al. 1997), which lack suitable uncertainties, nor the magnitudes $B_{J} R_{F} I_{N}$ from digitised photographic plates (e.g., USNO-B1; Monet et al. 2003). Besides, I have not taken into account the $J H K$ magnitudes provided by García-Lario et al. (1997) and Doering \& Meixner (2009). In Table 1 I give eleven accurate measurements of the $V$ magnitude of V1247 Ori, as published in the literature. The mean and standard deviation of all the measurements are $\bar{V}=$ 9.861 and $\sigma(V)=0.033 \mathrm{mag}$. With the information at the 18

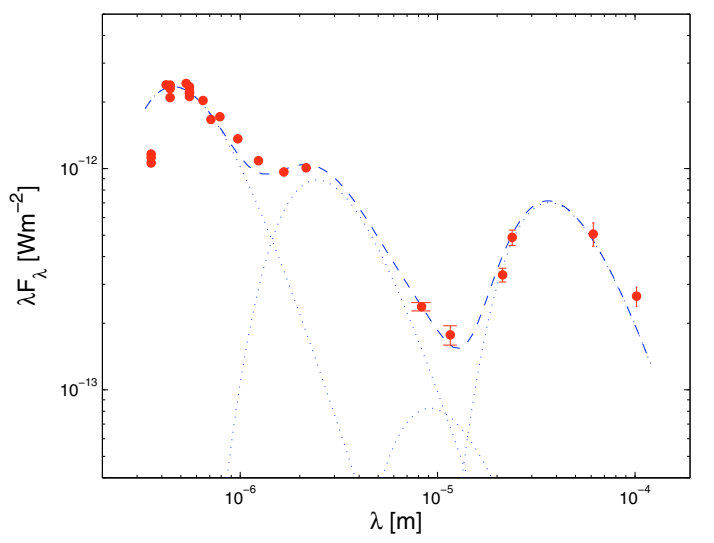

Fig. 2. Spectral energy distribution of V1247 Ori. Data points correspond, from left to right, to $U, B_{T}, B, V_{T}, V, R_{C}, R, I_{C}, I, J, H, K_{\mathrm{s}}$, and $8,12,21,25,60$, and $100 \mu \mathrm{m}$. Only as a guidance and without any fitting purpose, four spherical black bodies of $T_{\mathrm{eff}}=8000,1500,400$, and $100 \mathrm{~K}$ are plotted with dotted (blue) lines. The result of combining the four black bodies is marked with a dashed (blue) line.

wavelength bands in Table 2, I constructed the SED shown in Fig. 2. The infrared flux excess redwards of $1 \mu \mathrm{m}$ is obvious.

The light curves in Fig. 1 were built using only the ASAS data points corresponding to the aperture " 2 " (i.e., 4 pixels, or about 1 arcmin - Pojmański 2002; Caballero et al. 2010), which gave the lower mean photometric error. Besides, only data points with the best quality (grades "A" and "B") were used. The ASAS photometry of V1247 Ori was not affected by neighbouring bright stars (Fig. 3), and thus the observed photometric variability is intrinsic to the Herbig Ae/Be star. For a better visualisation of the occultation events, some captions of the light curve of V1247 Ori are shown in the four panels of Fig. 4. I implemented an iterative process to differentiate the data points that deviate more than $3 \sigma$ from a "cleaned" light curve with the rest of the data points. The mean and standard deviation of the "cleaned" light curve, indicated with small dots in Fig. 4, are $\bar{V}$ $=9.847 \mathrm{mag}$ and $\sigma(V)=0.024 \mathrm{mag}$, consistent with the values given in Table 1.

\section{Discussion}

The ASAS light curve of V1247 Ori has 509 data points and covers about nine years with short seasonal gaps. Only 51 data points, marked with open circles in Fig. 4, deviate more than $3 \sigma$ with respect to the "cleaned" light curve. I carried out a periodogram analysis on the other 458 data points of the "cleaned" light curve. The analysis, identical to the one in Caballero et al. (2010), gave a power maximum at $560 \mathrm{~d}$, which is an alias of the total time span (one sixth). The second most powerful peak is much narrower and falls at $P \approx 76.5 \mathrm{~d}$. It likely corresponds to the $P_{2} \approx 75.255 \mathrm{~d}$ periodicity found by Debosscher et al. (2007). Given the typical time interval between consecutive ASAS observations (about 2-3 d; see again Caballero et al. 2010), this analysis is not sensitive to the short $\delta$ Scuti periodicities of 2.16-2.33 h found by Lampens \& Rufener (1990) and Debosscher et al. (2007).

There are two kinds of outlier data points (circles in Fig. 4). Six of the 51 outliers are brighter than the "cleaned" light curve and are likely associated to flare-like events. There were three such events in the season 2005-2006, but only one in each of the following three seasons. The light curve hints at an increasing amplitude of the flare-like events with time, from about $0.10 \mathrm{mag}$ in 2006 to about $0.15 \mathrm{mag}$ in 2009 . They follow one after other 


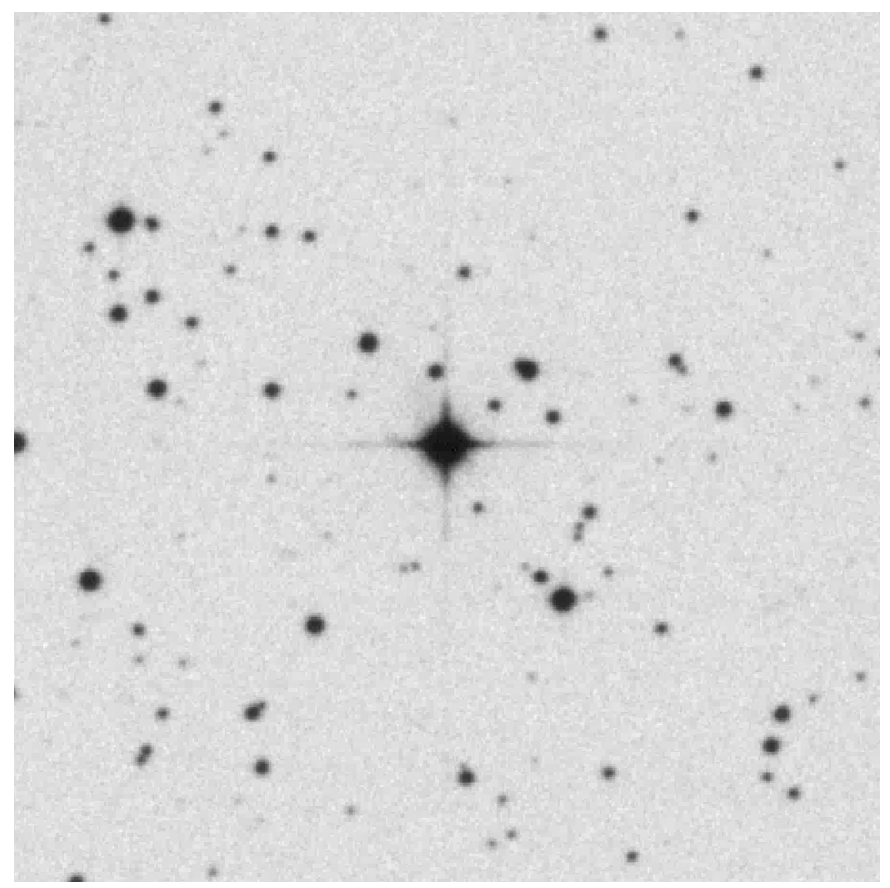

Fig. 3. Inverted-colour image centred on V1247 Ori from the SuperCOSMOS digitisations of the United Kingdom Schmidt Telescope photographic red plates. The size is $5 \operatorname{arcmin} \times 5 \operatorname{arcmin}$. North is up and east is to the left. The second and third brightest stars in the field have magnitudes $V=14.3 \pm 0.2$ (at 1.1 arcmin to the southwest of V1247 Ori) and $13.8 \pm 0.4 \mathrm{mag}$ (at $2.3 \mathrm{arcmin}$ to the northeast).

with about the same time interval of about one year (note that each "flare" is a single event that was observed only once per night). The light curve of the stable field star HD 290760 in Fig. 1 has the same standard deviation as the "cleaned" light curve of V1247 Ori, and shows three outlier data points (of a total of 459). From it, I estimate that only about $0.7 \%$ of the data points of the light curve of V1247 Ori can be affected by systematics of unknown origin and that thus most of the flare-like events can be real. They can be related to the $\mathrm{H} \alpha$ emission discovered by McConnell (1982) and probably to on-going accretion from the circumstellar disc of V1247 Ori. The other 45 outlier data points are fainter than the "cleaned" light curve. Most of them were associated to the main occultation events in 2002 Dec. and 2004 Feb., but there were also secondary events of less depth containing groups of up to four data points (e.g., 2001 Oct. [+0.20 mag], 2003 Feb. [+0.20 mag], 2003 Dec. [+0.35 mag], and 2008 Nov.-Dec. [+0.15 mag]). Besides, there was an isolated data point in 2005 Dec. that was about 0.65 mag fainter than the mean "cleaned" light curve.

The two main occultation events are different in shape (bottom panels in Fig. 4). On the one hand, the 2002 Dec. event lasted for about two weeks and was quite symmetric, with a pronounced drop and raise around the minimum on 2002 Dec. 20. Because of the different slopes of the drop and raise, the event could have been deeper than 1.65 mag on 2002 Dec. 21-23. On the other hand, the 2004 Feb. event was not so deep (+1.20 mag), lasted longer (about three weeks), and was highly asymmetric, with a series of local magnitude maxima and minima. While the origin of the first event might have been a transiting stellar companion, the one of the 2004 Feb. event must be associated to an heterogeneous envelope, which is likely the circumstellar disc (a sudden burst of cool spots in the photosphere of an early-type star would be rather speculative).
Table 2. Basic data of the Herbig Ae/Be star V1247 Ori.

\begin{tabular}{lcll}
\hline \hline Quantity & Value & Unit & Reference $^{a}$ \\
\hline$\alpha^{J 2000}$ & 053805.25 & & TYC \\
$\delta^{J 2000}$ & -011521.7 & & TYC \\
$\mu_{\alpha} \cos \delta$ & $-0.9 \pm 1.1$ & mas a $^{-1}$ & TYC \\
$\mu_{\delta}$ & $+1.3 \pm 1.1$ & mas a $^{-1}$ & TYC \\
$U$ & $10.29 \pm 0.01:$ & $\mathrm{mag}$ & Sh62 \\
$B$ & $10.20 \pm 0.01:$ & $\mathrm{mag}$ & Sh62 \\
$B_{T}$ & $10.18 \pm 0.03$ & $\mathrm{mag}$ & TYC \\
$V$ & $9.88 \pm 0.01:$ & $\mathrm{mag}$ & Sh62 \\
$V_{T}$ & $9.85 \pm 0.03$ & $\mathrm{mag}$ & TYC \\
$R$ & $9.68 \pm 0.01:$ & $\mathrm{mag}$ & Vi03 \\
$R_{C}$ & $9.61 \pm 0.05$ & $\mathrm{mag}$ & Fu02 \\
$I$ & $9.44 \pm 0.01:$ & $\mathrm{mag}$ & Vi03 \\
$I_{C}$ & $9.32 \pm 0.05$ & $\mathrm{mag}$ & Fu02 \\
$J$ & $8.88 \pm 0.03$ & $\mathrm{mag}$ & 2MASS \\
$H$ & $8.20 \pm 0.05$ & $\mathrm{mag}$ & 2MASS \\
$K_{\mathrm{s}}$ & $7.41 \pm 0.03$ & $\mathrm{mag}$ & 2MASS \\
$8 \mu \mathrm{m}$ & $0.66 \pm 0.03$ & $\mathrm{Jy}$ & MSX6C \\
$12 \mu \mathrm{m}$ & $0.68 \pm 0.07$ & $\mathrm{Jy}$ & IPAC \\
$21 \mu \mathrm{m}$ & $2.36 \pm 0.17$ & $\mathrm{Jy}$ & MSX6C \\
$25 \mu \mathrm{m}$ & $3.9 \pm 0.3$ & $\mathrm{Jy}$ & IPAC \\
$60 \mu \mathrm{m}$ & $10.4 \pm 1.2$ & $\mathrm{Jy}$ & IPAC \\
$100 \mu \mathrm{m}$ & $9.0 \pm 0.9$ & $\mathrm{Jy}$ & IPAC \\
Sp. type & A5 III, F0 Ve & & SC71, Vi03 \\
\hline & & &
\end{tabular}

Notes. ${ }^{(a)}$ TYC: Høg et al. (2000); Sh62: Sharpless (1962); Vi03: Vieira et al. (2003); Fu02: Fujii et al. (2002); 2MASS: Skrutskie et al. (2006); MSX6C: Egan et al. (2003) (8 and $21 \mu \mathrm{m}$-band measurements correspond to the SPIRIT III/Midcourse Space Experiment bands A, $8.28 \mu \mathrm{m}$ [6.8-10.8 $\mu \mathrm{m}$ ], and $E, 21.34 \mu \mathrm{m}$ [18.2-25.1 $\mu \mathrm{m}$ ]; IPAC: Joint IRAS Science Working Group 1988; SC71: Schild \& Cowley (1971).

From the SED in Fig. 2, I confirm that the disc of V1247 Ori has at least two components at different temperatures. The simplest model indicates that there is a warm $(T \sim 1500 \mathrm{~K})$ inner component and a cool $(T \sim 100 \mathrm{~K})$ outer one, with a dim third component at an intermediate temperature $(T \sim 400 \mathrm{~K})$ in between. Obviously, protoplanetary discs are not isothermal spheres, and the black bodies in Fig. 2 must be understood only as a guidance. A more comprehensive analysis, accounting for the disc inner rim, shadow region, and the disc atmosphere and surface beyond the flaring radius should be used to derive accurate disc properties (e.g., radius and height of the rim, surface density, total disc size, disc luminosity - Dullemond et al. 2001). The high flux at $20-60 \mu \mathrm{m}$ reveals a large physical size of the outer-disc component.

Although occultations by circumstellar discs of young stellar objects in general and of Herbig Ae/Be stars in particular are not rare (e.g., Bibo \& Thé 1991; Grinin et al. 1991; Herbst et al. 1994; Bertout 2000; Dullemond et al. 2003), the case of V1247 Ori sheds bright light on the topic for several reasons:

- The variability type of Herbig Ae/Be stars with occulting edge-on discs is called UX Orionis after the best-observed star displaying this sort of activity. V1247 Ori is as bright as UX Ori itself ( $V \approx 9.6 \mathrm{mag}$ ), and more than 4 mag brighter than the interesting eclipsed star KH 15D (Hamilton et al. 2005).

- The SED of V1247 Ori is well characterised. The two models of protoplanetary discs that best explain the UX Orionis phenomenon are based on the occultation by clumps of dust and gas that cross our line of sight. These hydrodynamics fluctuations may be located in the outer-disc regions or in the puffed-up inner rim (Dullemond et al. 2003). The second 
location favours self-shadowed discs with relatively weak far-infrared excess. The red IRAS colour [12] - [60] $\approx$ $3.0 \mathrm{mag}$ and strong far-infrared excess of V1247 Ori supports instead the first location.

- Most interestingly, the stability of the bright phase of V1247 Ori, at the level of 0.02 mag or less, indicates that the variability observed in the 2004 Feb. event must be exclusively ascribed to the occulting disc clumps and not to photospheric variations. Thus, the spatial structure of the hydrodynamic fluctuations in the inner rim or the outer disc (maybe spiral arm-like) can be derived from the shape of the light curve with an appropriate analysis.

However, there might be further complications, such as stellar multiplicity. The SED of V1247 Ori has a gap around about $15 \mu \mathrm{m}$, which is frequently considered as a signature of binarity in a young star (e.g., Espaillat et al. 2007). If the star is a binary, the structure of the circumstellar disc around the primary can differ from the puffed-up inner rim model. This complication would make it difficult to localise the dust clouds screening the star from the observer. In addition, following the ideas shown by Grinin \& Rostopchina (1996), the unusual photometric properties of V1247 Ori can be caused by the intermediate orientation of its circumstellar disc, between pole-on (corresponding to active UX Ori stars with two-peaked $\mathrm{H} \alpha$ profiles) and edge-on (corresponding to non-active Herbig Ae/Be stars with P Cygni $\mathrm{H} \alpha$ profiles and Algol-type activity like AB Aur). Further photometric monitoring of V1247 Ori is required to ascertain the structure of its disc.

Acknowledgements. I thank V. P. Grinin for his helpful referee report. I am an investigador Ramón y Cajal at the Centro de Astrobiología. This research made use of the SIMBAD, operated at Centre de Données astronomiques de Strasbourg, France, and NASA's Astrophysics Data System. Financial support was provided by the Universidad Complutense de Madrid and the Spanish Ministerio de Ciencia e Innovación under grants AyA2008-06423-C03-03 and AyA200800695.

\section{References}

Bertout, C. 2000, A\&A, 363, 984

Bibo, E. A., \& Thé, P. S. 1991, A\&AS, 89, 319
Caballero, J. A., \& Solano, E. 2008, A\&A, 485, 931

Caballero, J. A., Cornide, M., \& de Castro, E. 2010, AN, 331, 257

Debosscher, J., Sarro, L. M., Aerts, C., et al. 2007, A\&A, 475, 1159

Doering, R. L., \& Meixner, M. 2009, AJ, 138, 780

Droege, T. F., Richmond, M. W., Sallman, M. P., et al. 2006, PASP, 118, 1666 (VizieR on-line data catalogue: II/271A)

Dullemond, C. P., Dominik, C., \& Natta, A. 2001, ApJ, 560, 957

Dullemond, C. P., van den Ancker, M. E., Acke, B., et al. 2003, ApJ, 594, L47

Egret, D., Didelon, P., McLean, B. J., Russell, J. L., \& Turon, C. 1992, A\&A, 258, 217

Epchtein, N., de Batz, B., Capoani, L., et al. 1997, Msngr, 87, 27

Espaillat, C., Calvet, N., D’Alessio, P., et al. 2007, ApJ, 670, L135

Fujii, T., Nakada, Y., \& Parthasarathy, M. 2000, A\&A, 385, 884

García, J. R., Cebral, J. R., Scoccimarro, E. R. et al. 1995, A\&AS, 109, 201

García-Lario, P., Manchado, A., Pych, W., et al. 1997, A\&AS, 126, 479

Grinin, V. P., \& Rostopchina, A. N. 1996, ARep, 40, 171

Grinin, V. P., Kiselev, N. N., Chernova, G. P., Minikulov, N. Kh., \& Voshchinnikov, N. V. 1991, Ap\&SS, 186, 283

Guetter, H. H. 1981, AJ, 86, 1075

Hamilton, C. M., Herbst, W., Vrba, F. J., et al. 2005, AJ, 130, 1896

Handler, G. 1999, IBVS, 4817, 1

Heckmann, O. 1975, AGK 3. Star catalogue of positions and proper motions north of -2.5 deg declination, Hamburg-Bergedorf: Hamburger Sternwarte, ed. W. Dieckvoss (VizieR on-line data catalogue: I/61B)

Herbst, W., Herbst, D. K., Grossman, E. J., et al. 1994, AJ, 108, 1906

Høg, E., Fabricius, C., Makarov, V. V., et al. 2000, A\&A, 355, L27

Kazarovets, E. V., Samus, N. N., \& Goranskij, V. P. 1993, IBVS, 3840, 1

Lampens, P., \& Rufener, F. 1990, A\&AS, 83, 145

MacConnell, D. J. 1982, A\&AS, 48, 355

Monet, D. G., Levine, S. E., Canzian, B., et al. 2003, AJ, 125, 984

Nesterov, V. V., Kuzmin, A. V., Ashimbaeva, N. T., et al. 1995, A\&AS, 110, 367

Pojmański, G. 2002, AcA, 52, 397 (VizieR on-line data catalogue: II/264)

Richmond, M. W., Droege, T. F., Gombert, G., et al. 2000, PASP, 112, 397

Rodrigues, C. V., Sartori, M. J., Gregorio-Hetem, J., et al. 2009, ApJ, 698, 2031

Rodríguez, E., López de Coca, P., Rolland, A., Garrido, R., \& Costa, V. 1994, A\&AS, 106, 21

Rodríguez, E., López-González, M. J., \& López de Coca, P. 2000, A\&AS, 144, 469

Rufener, F. 1976, A\&AS, 26, 275

Rufener, F. 1988, Catalogue of stars measured in the Geneva Observatory photometric system, Sauverny: Observatoire de Genève

Schild, R. E., \& Cowley, A. P. 1971, A\&A, 14, 66

Sharpless, S. 1962, ApJ, 136, 767

Skrutskie, M. F., Cutri, R. M., Stiening, R., et al. 2006, AJ, 131, 1163

Vieira, S. L. A., Corradi, W. J. B., Alencar, S. H. P. et al. 2003, AJ, 126, 2971

Warren, W. H., Jr., \& Hesser, J. E. 1977, ApJS, 34, 115

Wouterloot, J. G. A., \& Walmsley, C. M. 1986, A\&A, 168, 237

Wouterloot, J. G. A., Walmsley, C. M., \& Henkel, C. 1988, A\&A, 203, 367

Wouterloot, J. G. A., Henkel, C., \& Walmsley, C. M. 1989, A\&A, 215, 131

Zwintz, K. 2008, ApJ, 673, 1088

Page 5 is available in the electronic edition of the journal at http://www . aanda.org 
J. A. Caballero: The occultation events of the Herbig Ae/Be star V1247 Ori
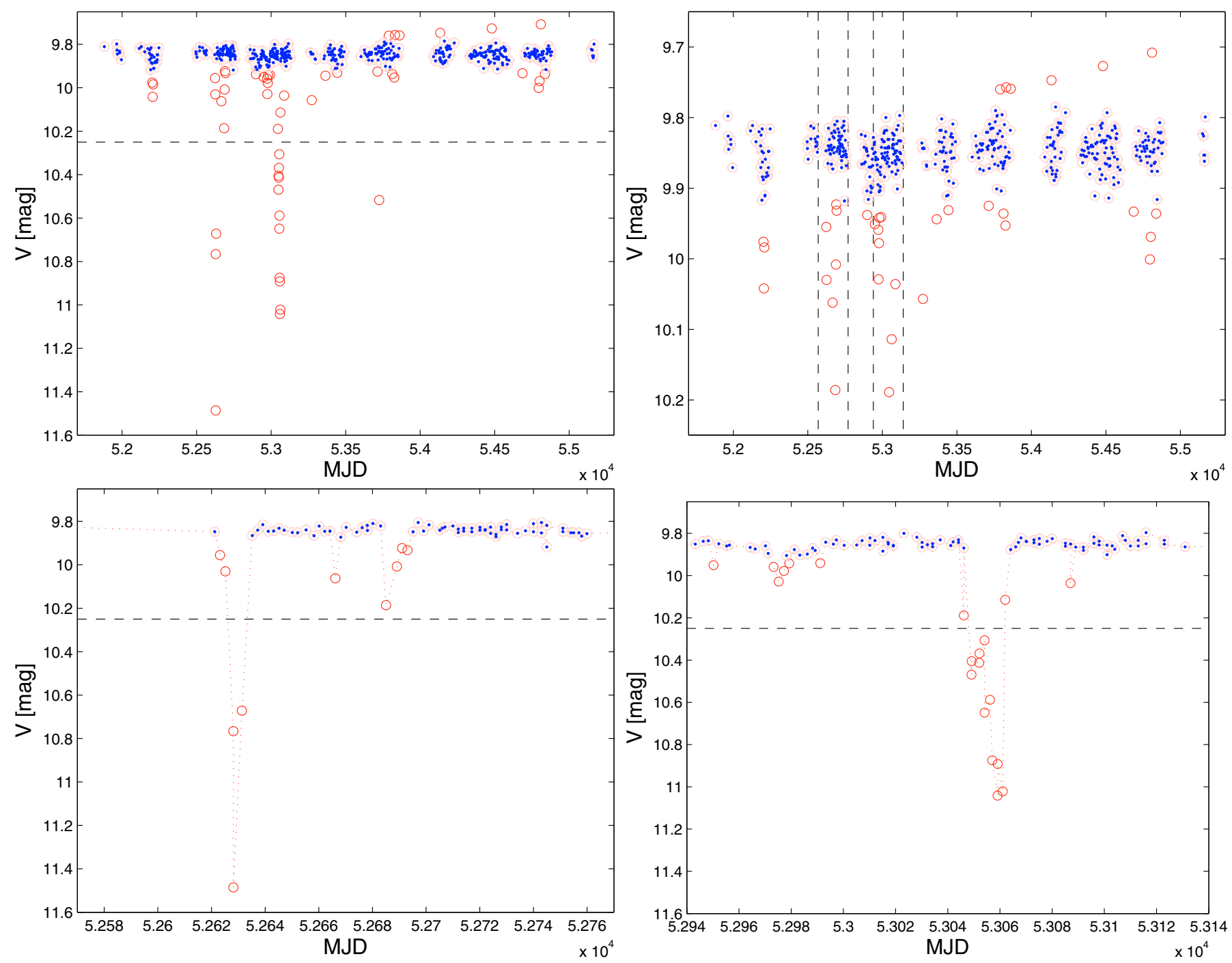

Fig. 4. Different captions of the ASAS light curve of V1247 Ori. Data points that deviate less and more than $3 \sigma$ from the iteratively-"cleaned" light curve are plotted with small (blue) dots and open (red) circles, respectively. Top left panel: full time and magnitude ranges. The horizontal dashed line marks the value $V=10.25 \mathrm{mag}$. Top right panel: full time range. Only magnitudes brighter than $V=10.25$ mag are shown. The vertical dashed lines indicate the enlargement areas in the bottom panels, corresponding to the two main occultation events. Bottom panels: full magnitude ranges, $200 \mathrm{~d}$-wide time intervals around the 2002 Dec. (left) and 2004 Feb. (right) occultation events. 